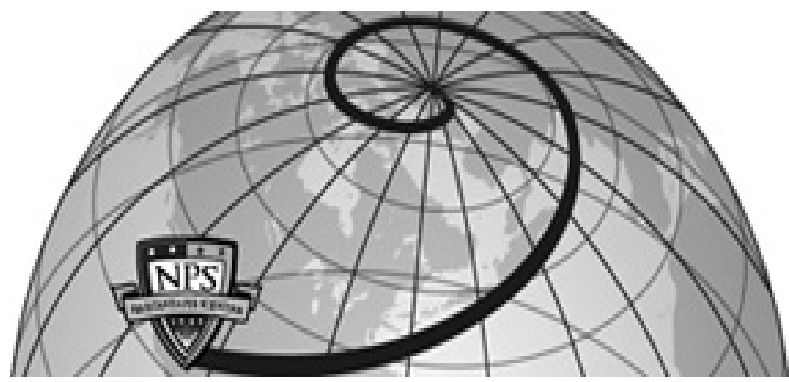

Calhoun: The NPS Institutional Archive DSpace Repository

\title{
Uniform representation of data types in Polymorphic C
}

Pederson, Carl M.

Monterey, California. Naval Postgraduate School

https://hdl.handle.net/10945/24399

Downloaded from NPS Archive: Calhoun

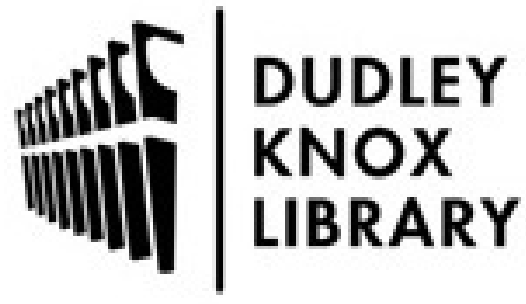

http://www.nps.edu/library
Calhoun is the Naval Postgraduate School's public access digital repository for research materials and institutional publications created by the NPS community. Calhoun is named for Professor of Mathematics Guy K. Calhoun, NPS's first appointed -- and published -- scholarly author.

Dudley Knox Library / Naval Postgraduate School 411 Dyer Road / 1 University Circle Monterey, California USA 93943 


\section{NAVAL POSTGRADUATE SCHOOL Monterey, California}
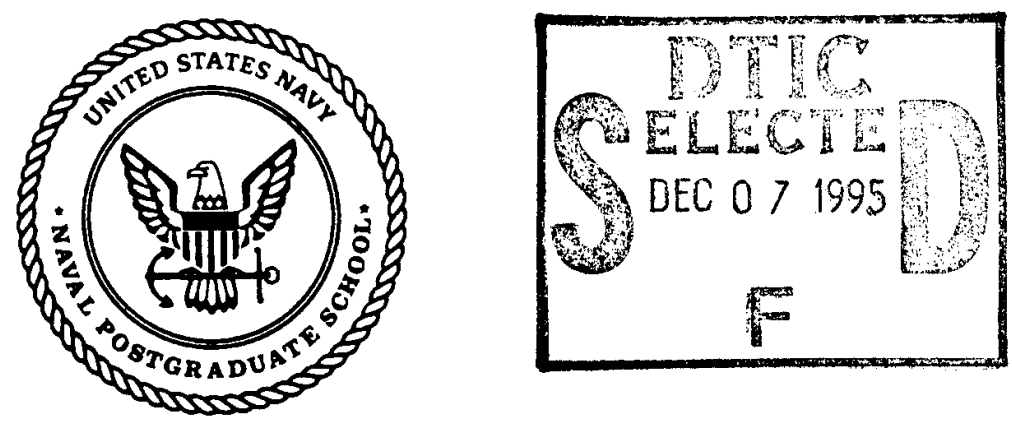

\section{UNIFORM REPRESENTATION OF DATA TYPES IN POLYMORPHIC C}

by

Carl M. Pederson, Jr., CDR, USN

October 1995

Approved for public release; distribution is unlimited

Prepared for: Naval Postgraduate School

Monterey, CA 93943 


\section{NAVAL POSTGRADUATE SCHOOL \\ Monterey, California}

Rear Admiral M. J. Evans

Richard Elster

Superintendent

Provost

This report was prepared for a directed study course (CS 4800) titled Advanced Topics in Compilation.

Reproduction of all or part of this report is authorized.

Carmipelemon.

CDR Carl M. Pederson, Jr., USN
Reviewed by:

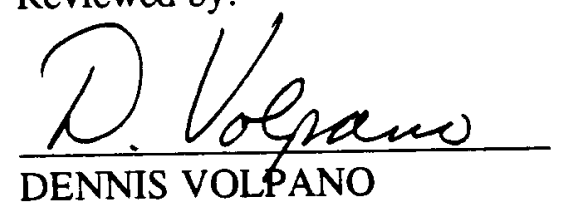

Assistant Professor

of Computer Science

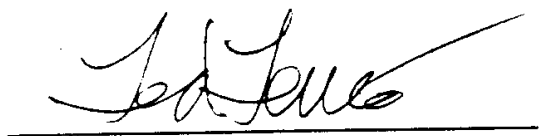

TED LEWIS

Chairman

Department of Computer Science
Released by:

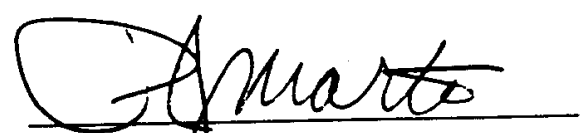

PAUL J. MARTO

Dean of Research

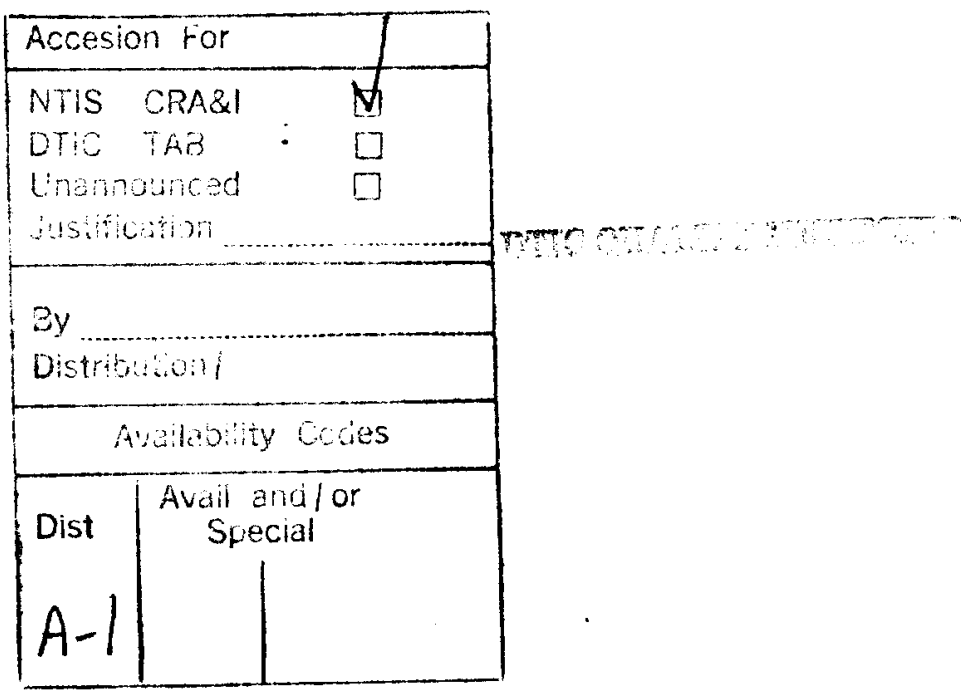


UNCLASSIFIED

SECURITY CLASSIFICATION OF THIS PAGE

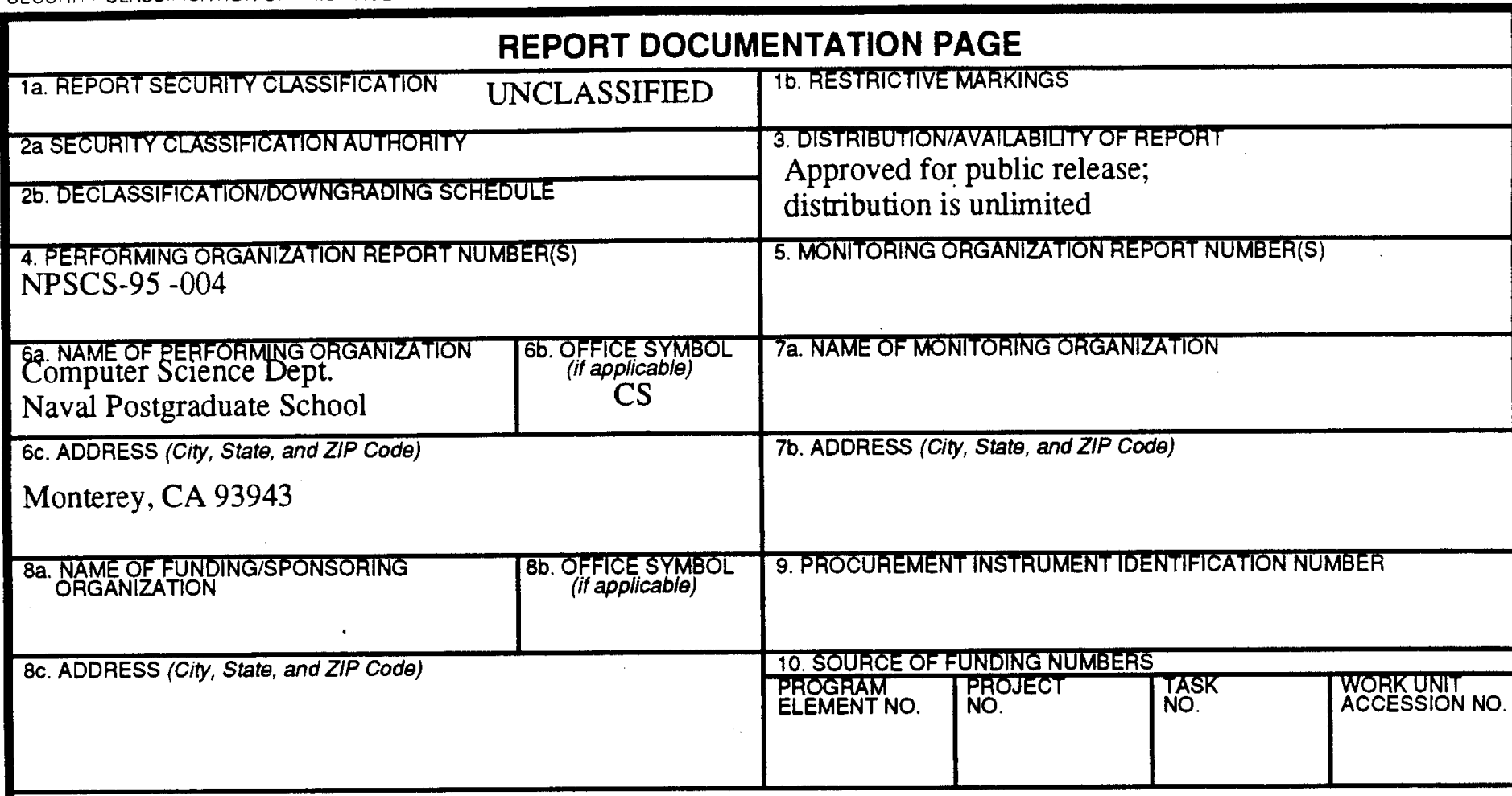

11. TITLE (Include Security Classification)

Uniform Representation of Data Types in Polymorphic C.

12. PERSONA AUTHOR(S)

CDR Carl M. Pederson Jr., USN

\begin{tabular}{l|l|l|l|l}
\hline $\begin{array}{l}\text { 3a. TYPE OF REPORT } \\
\text { Final }\end{array}$ & $\begin{array}{l}\text { 13b. TIME COVERED } \\
\text { FROM 7/95 TO 10/95 }\end{array}$ & $\begin{array}{l}\text { 14. DATE OF REPOAT (Year, Month, Day) } \\
\text { October } 1995\end{array}$ & PAGE COUNT \\
\hline
\end{tabular}

16. SUPPLEMMENTARYNOTATION

\begin{tabular}{|l|l|l|}
\hline 17. & \multicolumn{3}{|c|}{ COSATI CODES } \\
\hline FIELD & GROUP & SUB-GROUP \\
\hline & & \\
\hline & & \\
\hline
\end{tabular}

18. SUBJECT TERMS (Continue on reverse if necessary and identify by block number) polymorphism, $\mathrm{C}$ programming language

19. ABSTRACT (Continue on reverse if necessary and identify by block number)

A polymorphic dialect of $\mathrm{C}$, called Polymorphic C, has been proposed. The dialect retains the flexibility of $\mathrm{C}$ while incorporating ML-style polymorphism and rigorous type reconstruction. Supporting polymorphism in a programming language often requires sacrificing either speed, space, or both in the executable code. The preferred implementation of Polymorphic $\mathrm{C}$ would preserve the speed and space efficiency of $\mathrm{C}$. This paper demonstrates an approach for generating efficient executable code for Polymorphic $\mathrm{C}$ based on a variation of uniform representation and using byte-wise manipulation.

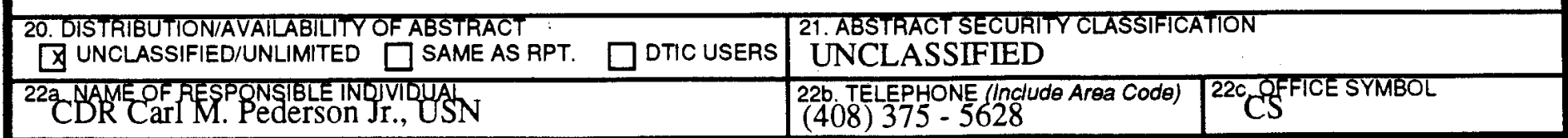




\section{Uniform Representation of Data Types in Polymorphic C}

\section{Abstract}

A polymorphic dialect of $\mathrm{C}$, called Polymorphic $C$, has been proposed. The dialect retains the flexibility of $\mathrm{C}$ while incorporating ML-style polymorphism and rigorous type reconstruction. Supporting polymorphism in a programming language often requires sacrificing either speed, space, or both in the executable code. The preferred implementation of Polymorphic $\mathrm{C}$ would preserve the speed and space efficiency of $\mathrm{C}$. This paper demonstrates an approach for generating efficient executable code for Polymorphic $\mathrm{C}$ based on a variation of uniform representation and using byte-wise manipulation.

\section{Introduction}

Providing the widely used imperative language $C$ with polymorphism could dramatically increase the reuse potential of $\mathrm{C}$ programs. A polymorphic dialect of $\mathrm{C}$, called Polymorphic $C$ (abbreviated Poly $C$ in this paper) has been proposed by Smith and Volpano [SmV095]. The dialect retains the flexibility of C while incorporating ML-style polymorphsim and rigorous type reconstruction. The acceptance of the dialect requires an efficient implementation. The compiled code must come close to the speed and space efficiency of $\mathrm{C}$.

Incorporating polymorphism in a programming language is generally incompatible with fast execution speed and low space overhead. Additionally polymorphism introduces various problems into the process of generating intermediate code during compilation. Many implementation techniques addressing these problems have been proposed for functional languages [MDCB91], [Ler92], [ShAp95], [Thi95]. These approaches could be implemented in a compiler for Poly $\mathrm{C}$, but not without adversely affecting executable code. This is because function calls are used to perform explicit coercion between monomorphic types and a uniform representation used by polymorphic functions (wrap 
and unwrap). This paper describes an implementation approach for Poly $C$ that preserves the speed efficiency with only a modest storage penalty.

The proposed implementation employs $\mathrm{C}$ code as the intermediate language. $\mathrm{A}$ monomorphic program is translated directly to $\mathrm{C}$ code. For polymorphic functions, a uniform and strict calling convention is used to allow different data types to be passed and returned from polymorphic functions. The proposed compilation approach is possible in Poly $\mathrm{C}$ because of its close correspondence with Kernighan and Ritchie $\mathrm{C}$ and its robust type system. The type inference system of Poly $\mathrm{C}$ can be used to determine which functions are polymorphic. Poly C's type system assures type correctness of a Poly C program. Intermediate code generation can be based on type information determined during the type checking process.

The specific assumptions necessary for the approach proposed in this paper are:

- the compiler can determine if a function is monomorphic or polymorphic,

- the compiler can ascertain information required by each polymorphic function (array size, element size, pointer arithmetic to apply, etc.) and is able to supply the information.

The details of the approach are described in the next section. Both the concepts and benefits of the approach are presented. In Section 3, examples are used to demonstrate the technique.

\section{Description of Approach}

There are four basic kinds of functions in an imperative language such as Poly $\mathrm{C}$ :

- monomorphic applicative -- a monomorphic function executed for its value,

- monomorphic imperative -- a monomorphic function executed for its effect and perhaps a value,

- polymorphic applicative -- a polymorphic function executed for its value,

- polymorphic imperative -- a polymorphic function executed for its effect and perhaps a value.

The first two kinds of functions when written in Poly $\mathrm{C}$ can be translated directly into $\mathrm{C}$ code. Polymorphic functions must be handled differently to allow the same code to be 
executed for all data types and produce correct results. This paper concentrates on intermediate code generation for the two kinds of polymorphic functions. To obtain executable code, each of the two kinds of polymorphic functions must be handled differently during compilation.

To correctly generate intermediate code from a Poly $\mathrm{C}$ program, the compiler must first determine the kind of function being translated. This is possible with information produced by Poly C's type inference system. Specifically,

- If the return type of the function is quantified (non-specialized) then the function is polymorphic.

- If the return type of the function is specialized then the function is monomorphic.

- If the function has no return type then it is executed for its effect.

A standard convention is always used to pass data to and from a polymorphic function. We use a calling convention employing a uniform representation. The types of the called function's actual parameters are ignored. The same number of bytes are allocated on the function's activation stack for each parameter regardless of its type. ${ }^{1}$ In the context of this paper, uniform representation refers to the notion that parameters occupy a uniform size on a polymorphic function's stack.

For example consider the polymorphic identity function, called id, written in Poly $\mathrm{C}$ and the associated intermediate code present below in Figure 1.

\begin{tabular}{|l|l|}
\hline \multicolumn{1}{|c|}{ Poly C Program } & \multicolumn{1}{c|}{ Intermediate Code } \\
\hline $\begin{array}{l}\text { letvar one := } \\
\text { let id }=\lambda x . x \text { in /polymorphic function id } \\
\text { in id }(1)\end{array}$ & long poly_id(long $x$ ) \{return $x ;$ \} // function id \\
& $\begin{array}{l}\text { int main() } \\
\text { int one }=\text { poly_id(1); } \\
\text { return } 0 ;\end{array}$ \\
\hline
\end{tabular}

Figure 1. Polymorphic Identity Function.

\footnotetext{
${ }^{1}$ The calling convention of typical $\mathrm{C}$ compilers passes arguments to functions on the stack and passes a return value from the function in a specific register(s).
} 
A default size of four bytes (type long) is used in the intermediate code for id's argument and return-value. The actual default size chosen for the implementation depends of the target architecture. In this paper, type long is used in the intermediate code for all examples of polymorphic functions. After compilation, the $\mathrm{C}$ program executes correctly for all data types currently supported in Poly C. Test results of the associated intermediate code for the Poly C program of Figure 1 are given in Appendix A.

As shown above, when a polymorphic function is executed for its value, an implementation using a calling convention employing default size for formal parameters on the activation stack is sufficient to produce correct results. However if a polymorphic function is executed for its effect, a different approach and additional information is required. The intermediate code generated performs a byte-by-byte manipulation of the effected store. To do this correctly the size (number of bytes) of actual parameters must be passed to the function. In this situation the compiler must be able to determine the size of the data type being passed to the function. The assignment function presented below in Figure 2 is an example of byte-by-byte copy.

\begin{tabular}{|c|c|}
\hline Poly C Program & Intermediate Code \\
\hline $\begin{array}{l}\text { let assign }=\lambda 1 \text { __side, } r \text { _side } .\left({ }^{*} \text { __side }:=r_{-} \text {side }\right) \\
\text { in } . . .\end{array}$ & $\begin{array}{l}\text { void poly_assign }\left(\text { char }{ }^{*} \text { I_side, char }{ }^{*} \text { __side, int }\right. \\
\text { size }) \\
\text { int } i=0 \text {; } \\
\text { for }(; i<\text { size } ; i++) \\
\quad *\left(1 \_ \text {side }+i\right)={ }^{*}\left(r_{-} \text {side }+i\right)\end{array}$ \\
\hline
\end{tabular}

Figure 2. A Polymorphic Function Executed for its Effect.

The intermediate code uses a type char pointer to facilitate byte-wise manipulation. The for loop copies each byte of actual parameter $r_{\text {s }}$ side to the appropriate byte of actual parameter $l_{\text {_side. }}$. The loop exit condition is based on the size of data type being manipulated. Test results of the associated intermediate code for the Poly $\mathrm{C}$ program of Figure 2 are given in Appendix B. 
Another situation that occurs with polymorphic functions is that a specialized function may need to be passed to a polymorphic function. As in the case of a polymorphic sort function, the comparison function specific to the data types being sorted, must be supplied.

As currently described, only three data types are of concern for polymorphic function calls in Poly C: integers, pointers, and arrays. However array names function as constant pointers as in $\mathrm{C}$, so really only two data types must be considered. In this paper various data types are used in the examples. The types are chosen to provide a range of store size and interpretation to sufficiently demonstrate the proposed implementation approach.

The primary advantage of this approach is that only polymorphic functions are affected by the proposed implementation. The impact on speed and storage of a Poly C program is minimal. Most of a Poly C's data type representation is unrestricted allowing storage optimization for all data values except parameters and return values of polymorphic functions. The only significant slow down potentially occurs when byte-wise manipulation is used in polymorphic functions executed for effect.

\section{Demonstration of Approach}

This section demonstrates the viability of implementing Poly $\mathrm{C}$ using the ideas presented above (default size occupied on the stack and byte-wise copy). Various conditions related to polymorphic functions that may occur in a Poly $\mathrm{C}$ program are explored. These conditions involve parameter passing combinations, functions executed for their value and functions executed for their effect, and continuation combinations ${ }^{2}$.

\footnotetext{
${ }^{2}$ Chained function calls such as $\mathrm{f} 1(\mathrm{f} 2(\mathrm{f} 3(\mathrm{x})))$.
} 
In [MDCB91] four combinations of parameter passing encountered in polymorphism are discussed.

1. A concrete (specialized) actual parameter passed to a concrete formal parameter.

2. A concrete actual parameter passed to a quantified (non-specialized) formal parameter.

3. A quantified actual parameter passed to a concrete formal parameter.

4. A quantified actual parameter passed to a quantified formal parameter.

For the approach presented in this paper, these cases are not of primary interest since in Poly $\mathrm{C}$ all actual parameters are concrete ${ }^{3}$.

A parameter passing combination that is of interest is the situation where a function is passed as a parameter to a polymorphic function. In the example given below in Figure 3 a polymorphic function called apply is passed two parameters, a data value and a function. The actual parameter for the function is passed via a pointer. Test results of the associated intermediate code for the Poly $\mathrm{C}$ program of Figure 3 are given in Appendix C.

\begin{tabular}{|c|c|}
\hline Poly C Program & Intermediate Code \\
\hline $\begin{array}{l}\text { letvar succ_of_x }= \\
\text { let apply }=\lambda x, \lambda f . f x \text { in } \\
\text { let int_succ }=\lambda \mathrm{a} . \mathrm{a}+1 \\
\text { in apply(2, int_succ) }\end{array}$ & $\begin{array}{l}\text { long poly_apply(long } x, \text { void }\left({ }^{*} \text { fun }\right)(0)\{\text { return fun }(x) ;\} \\
\text { int int_succ(int a) (retum a }+1 ;\} \\
\text { int main }() \\
\text { int } x=2 \text {; } \\
\text { int succ_of_x; } \\
\text { succ_of_ } x=\text { poly_apply(x, int_succ); } \\
\text { return } 0 \text {; }\end{array}$ \\
\hline
\end{tabular}

Figure 3. A Function Passed as a Parameter to a Polymorphic Function.

The examples given in Figure 1 and Figure 3 involved functions executed for their value. When a function is executed for its effect a byte-wise copy is used to change the

\footnotetext{
${ }^{3}$ A polymorphic function passed as an actual parameter is concrete and has type pointer.
} 
content of the appropriate memory locations. In this situation, the size of the data value being manipulated must be passed to the polymorphic function. This was demonstrated in the assignment example in the previous section (Figure 2).

The continuation of function calls (chaining of functions) can occur in four combinations.

1. A monomorphic function calls a monomorphic function.

2. A monomorphic function calls a polymorphic function.

3. A polymorphic function calls a monomorphic function.

4. A polymorphic function calls a polymorphic function.

The first calling combination does not involve any polymorphic functions and is not demonstrated. Also combination number three, a polymorphic function (apply) calls a monomorphic function (int_succ) was demonstrated above in Figure 3. Examples for each of the other two combinations are given below in Figure 4 and Figure 5. The Poly $\mathrm{C}$ program in Figure 5 is from [ $\mathrm{SmV095].} \mathrm{Test} \mathrm{results} \mathrm{of} \mathrm{the} \mathrm{associated} \mathrm{intermediate} \mathrm{code}$ for the Poly $C$ programs of Figure 4 and Figure 5 are given in Appendix D and Appendix E respectively.

\begin{tabular}{|c|c|}
\hline Poly C Program & Intermediate Code \\
\hline $\begin{array}{l}\text { letvar } a=1234 \text { in } \\
\text { letvar copy_of_a }=0 \text { in } \\
\text { let id }=\lambda x . x \text { in } \\
\text { let int_id }=\lambda x \text {.id(x) in } \\
\text { copy_of_a }:=\text { int_id(a) }\end{array}$ & $\begin{array}{l}\text { // polymorphic id function } \\
\text { long poly_id(long } x \text { ) \{return } x ;\} \\
\text { // integer id function calls poly_id } \\
\text { int int_id(int } x) \text { \{return poly_id(x); } \\
\text { int main() } \\
\text { int a = 1234; } \\
\text { int copy_of_a =0; } \\
\text { copy_of_a = int_id(a); } \\
\text { retur 0; }\end{array}$ \\
\hline
\end{tabular}

Figure 4. A Monomorphic Function Calls a Polymorphic Function. 


\begin{tabular}{|c|c|}
\hline Poly C Program & Intermediate Code \\
\hline 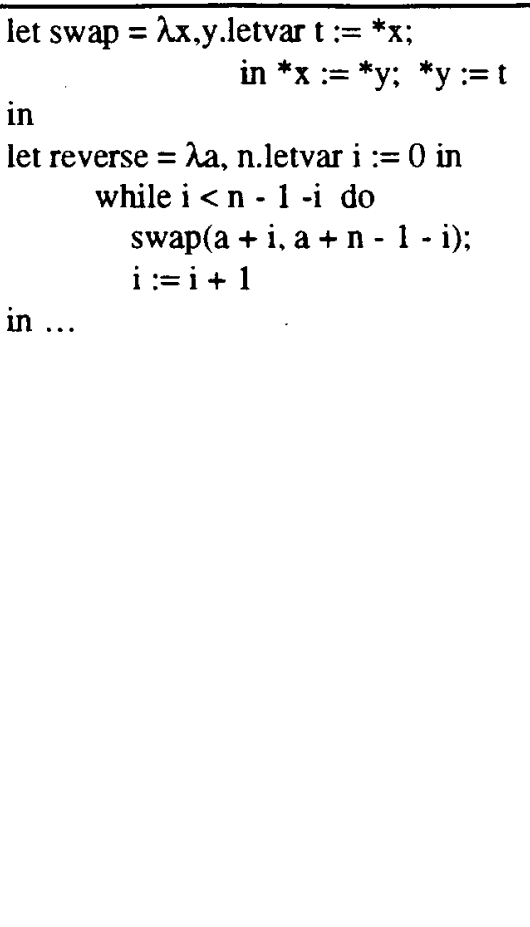 & 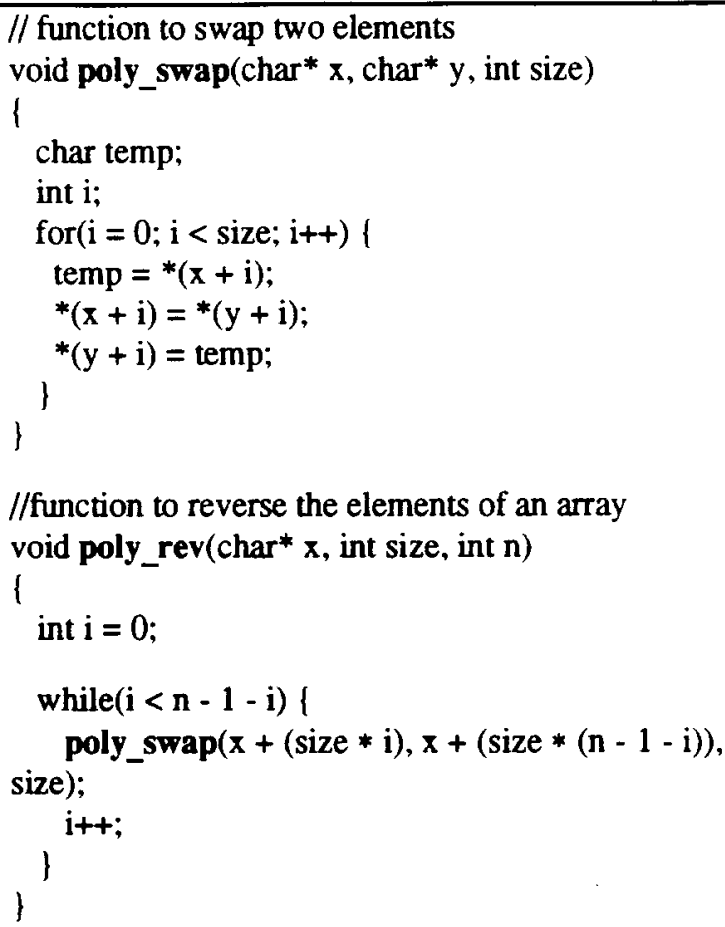 \\
\hline
\end{tabular}

Figure 5. A Polymorphic Function Calls a Polymorphic Function.

\section{Conclusions}

This paper demonstrated that intermediate $C$ code can be used to efficiently implement polymorphism in Poly C.

There is still much to be done related to the approach presented in this paper.

- A translation scheme must be developed.

- Comparing the efficiency of this approach with other approaches.

- The method will need to be extended to more complicated data types as they are include in Poly C.

Although a translation scheme was not provided, the examples presented in Section 3 can serve as a guide for the development of formal methods for generating intermediate code. Various implementation methods for Poly C ([Bon95], the approach 
presented in this paper, etc.) should be benchmarked for speed and space efficiency and compared with each other. From the perspective of intermediate code generation, extending Poly $\mathrm{C}$ to include more complicated data types does not appear to be an issue. The results of the examples presented in the appendices demonstrate how pointers can be used to handle other data types such as floats and structures.

One issue is the warnings generated by the compiler when the intermediate code is compiled. The type checking done by the $\mathrm{C}$ compiler when generating the executable program gives type warnings that can be ignored for the most part. Translating directly to assembly code would alleviate the problem. 


\section{APPENDIX A}

\section{Polymorphic Identity Function}

\section{Executable Code}

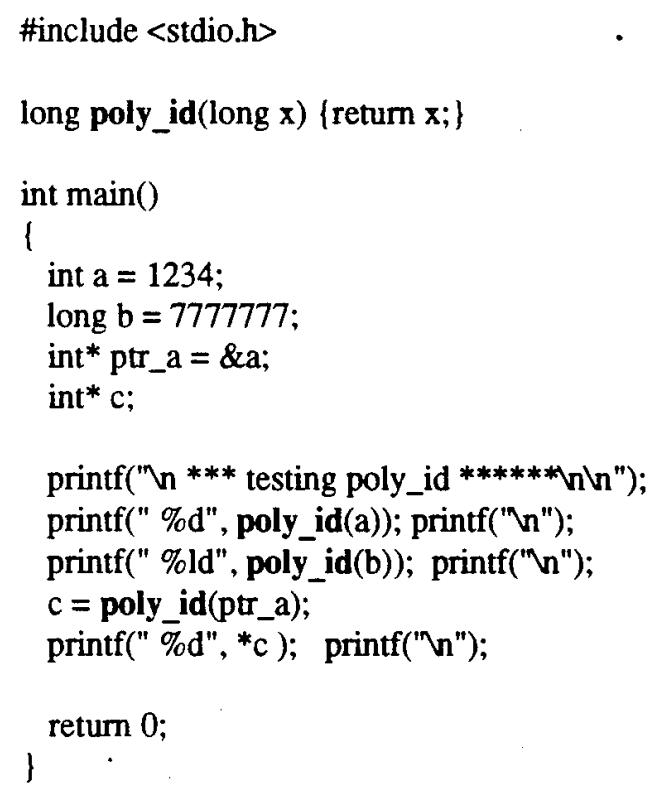

\section{$\underline{\text { Test Results }}$}

*** testing poly_id $* * * * * *$

1234

7777777

1234

\section{Waming}

line number: (15) : warning: 'argument' : different levels of indirection line number: (15) : warning: 'poly_id' : different types for formal and actual parameter 1 line number: (15) : warning: ' $=$ ' : different levels of indirection .

line number: (15): warning: conversion of near pointer to long integer 



\section{APPENDIX B}

\section{Polymorphic Assignment Function}

\section{Executable Code}

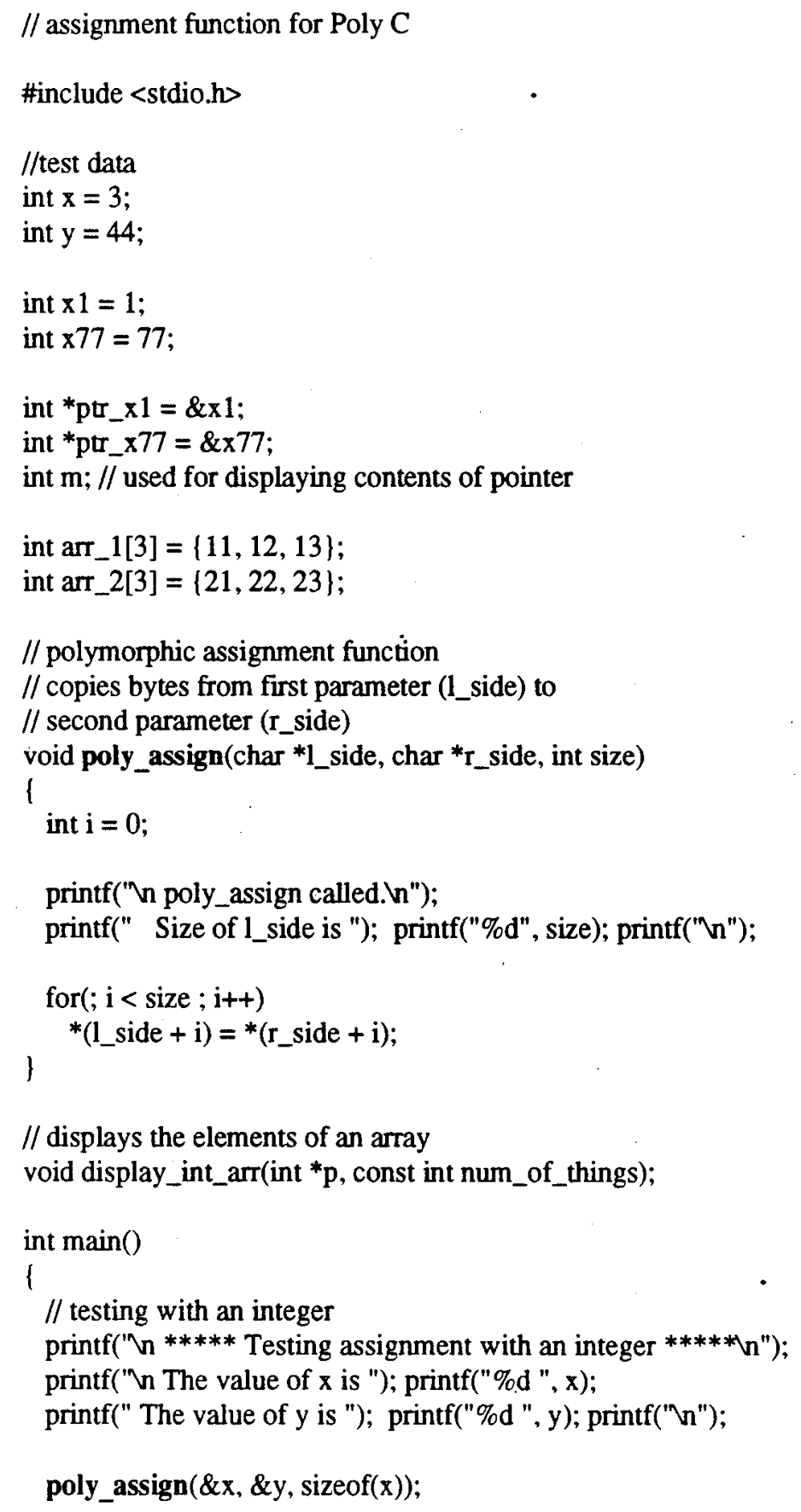




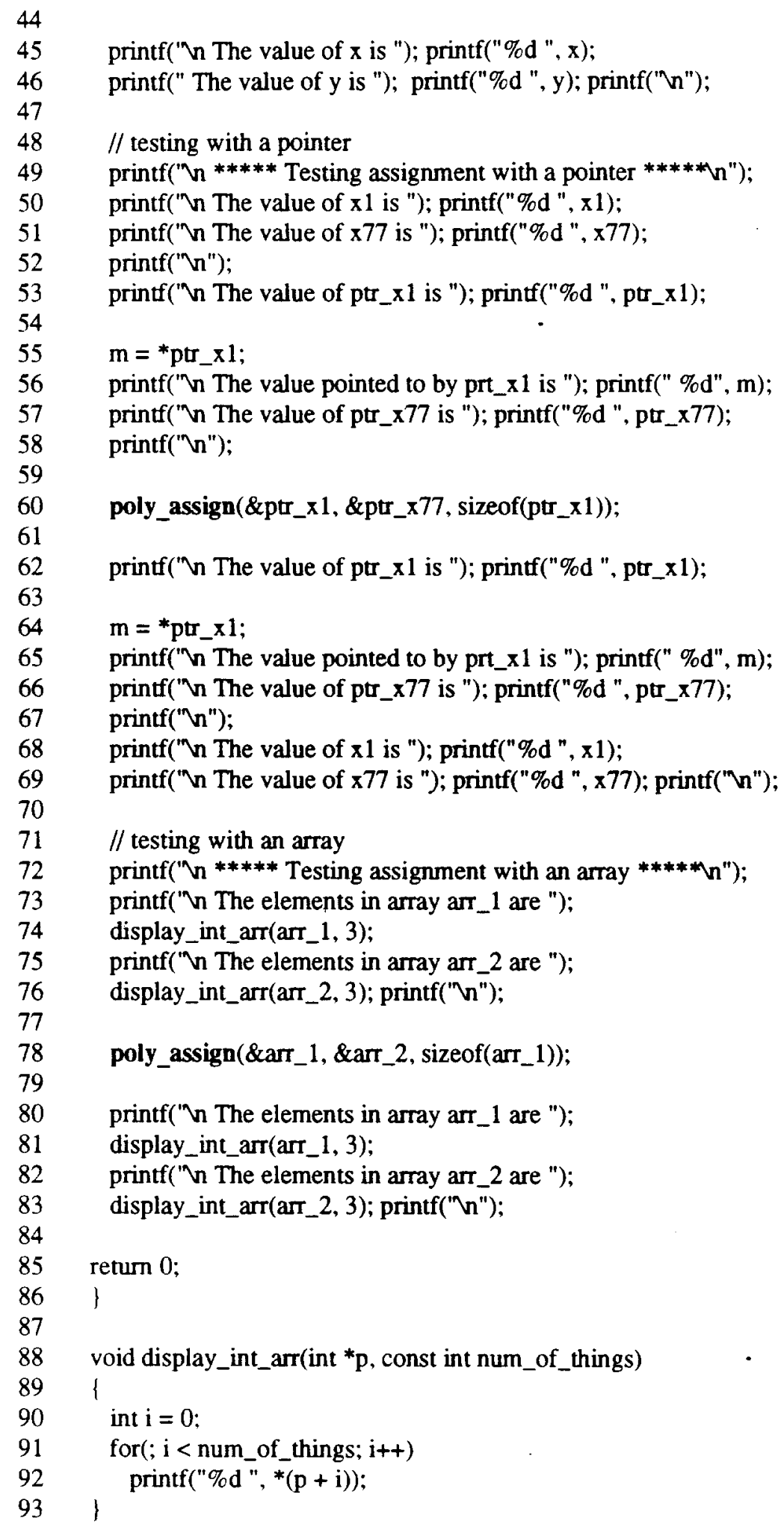

printf(" $\mathrm{n}$ The value of $\mathrm{x}$ is "); printf("\%d ", $\mathrm{x})$;

printf(" The value of $y$ is "); printf("\%d ", y); printf(" $n ")$;

$/ /$ testing with a pointer

printf(" $\vee$ ***** Testing assignment with a pointer ***** $(n ")$;

printf(" $\mathrm{n}$ The value of $\mathrm{x} 1$ is "); printf("\%d ", $\mathrm{x} 1$ );

printf(" $n$ The value of $x 77$ is "); printf("\%d ", $x 77)$;

printf("ท");

printf(" $n$ The value of ptr_x1 is "); printf("\%d ", pt__1);

$\mathrm{m}={ }^{*} \mathrm{ptr} \_\mathrm{xl}$;

printf("Vn The value pointed to by prt_x1 is "); printf(" \%d", m); printf(" $\mathrm{n}$ The value of ptr_x77 is "); printf("\%d ", ptr_x77);

printf("n");

\section{poly_assign(\&ptr_x1, \&ptr_x77, sizeof(ptr_x1));}

printf(" $n$ The value of ptr_xl is "); printf("\%d ". ptr_xl);

$\mathrm{m}={ }^{*} \mathrm{ptr} \times \mathrm{x1}$;

printf(" $\vee$ The value pointed to by prt_x 1 is "); printf(" \%d", m); printf("V The value of ptr_x77 is "); printf("\%d ",ptr_x77); printf("ทn");

printf(" $n$ The value of $x 1$ is "); printf("\%d ", $x 1)$;

printf(" $n$ The value of $x 77$ is "); printf("\%d ", $x 77)$; printf(" $\vee n ") ;$

// testing with an array printf(" $n$ ***** Testing assignment with an array ***** $n ")$; printf(" $\mathrm{V}$ The elements in array arr_1 are "); display_int_arr(arr_1, 3); printf(" $n$ The elements in array arr_2 are "); display_int_arr(arr_2, 3); printf(" $n$ ");

poly_assign(\&arr_1, \&arr_2, sizeof(arr_1));

printf(" $n$ The elements in array arr_1 are "); display_int_arr(art_1, 3);

printf(" $\mathrm{n}$ The elements in array arr_2 are "); display_int_arr(arr_2, 3); printf(" $n$ ");

return 0;

I

void display_int_arr(int *p, const int num_of_things)

1

int $\mathrm{i}=0$;

for(; i < num_of_things; $i++)$

printf("\%d ", * $(p+i))$; 
Test Results

***** Testing assignment with an integer *****

The value of $x$ is 3 The value of $y$ is 44

poly_assign called.

Size of 1_side is 2

The value of $\mathrm{x}$ is 44 The value of $\mathrm{y}$ is 44

***** Testing assignment with a pointer $* * * * *$

The value of $x 1$ is 1

The value of $x 77$ is 77

The value of ptr_ $x 1$ is 20

The value pointed to by prt_x 1 is 1

The value of ptr_x 77 is 22

poly_assign called.

Size of 1_side is 2

The value of $p t r \_x 1$ is 22

The value pointed to by prt_x 1 is 77

The value of ptr_x 77 is 22

The value of $x 1$ is 1

The value of $x 77$ is 77

***** Testing assignment with an array *****

The elements in array arr_1 are 111213

The elements in array arr_2 are 212223

poly_assign called.

Size of 1_side is 6

The elements in array arr_1 are 212223

The elements in array arr_2 are 212223

\section{Warnings}

line number: (43) : warning: 'argument' : indirection to different types line number: (43) : warning: 'argument' : indirection to different types line number: (60) : warning: 'argument' : different levels of indirection line number: (60) : warning: 'poly_assign' : different types for formal and actual parameter 1 line number: (60) : warning: 'argument' : different levels of indirection line number: (60) : warning: 'poly_assign' : different types for formal and actual parameter 2 line number: (78) : warning: 'argument' : different levels of indirection line number: (78) : warning: 'poly_assign' : different types for formal and actual parameter 1 line number: (78) : warning: 'argument' : different levels of indirection line number: (78) : warning: 'poly_assign' : different types for formal and actual parameter 2 
$$
-
$$ 


\section{APPENDIX C}

\section{Monomorphic Function Passed as a Parameter to a Polymorphic Function}

\section{$\underline{\text { Executable Code }}$}

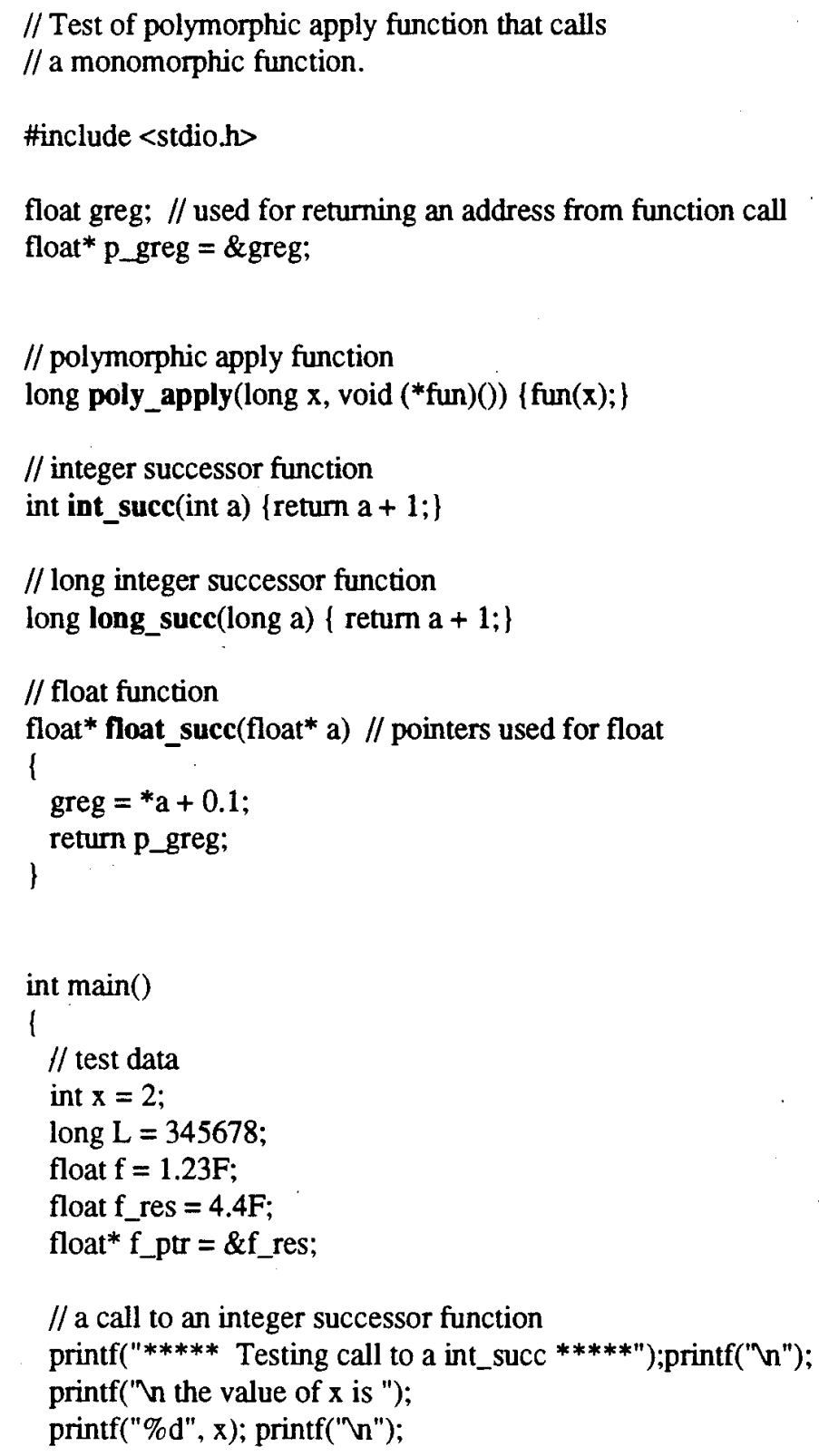


printf(" the value of poly_apply(x, int_succ) is ");

printf("\%d", poly_apply(x, int_succ)); printf(" $v \backslash n ") ;$

$/ / \mathrm{a}$ call to a long integer successor function printf("***** Testing call to a long_succ *****");printf("v"); printf(" $n$ the value of $L$ is "); printf("\%ld", L); printf("Vn"); printf(" the value of poly_apply(L, long_succ) is "); printf("\%ld", poly_apply(L, long_succ)); printf("Vinn");

// a call to a float successor function printf("***** Testing call to a float_succ *****");printf(" $\mathrm{n}$ "); printf(" $v$ the value of $f$ is "); printf("\%f", f); printf("v"); printf(" the value of poly_apply(f, float_succ)"

" $n$ [incremented by 0.1$]$ is "); f_ptr = poly_apply (\&f, float_succ); $\mathrm{f}_{\text {_res }}={ }^{*} \mathrm{f} \_\mathrm{ptr}$; printf("\%f", f_res); printf("ทnı");

return 0 ; )

\section{$\underline{\text { Test Results }}$}

***** Testing call to a int_succ *****

the value of $x$ is 2

the value of poly_apply(x, int_succ) is 3

***** Testing call to a long_succ *****

the value of $\mathrm{L}$ is 345678

the value of poly_apply(L, long_succ) is 345679

***** Testing call to a float_succ *****

the value of $f$ is 1.230000

the value of poly_apply(f, float_succ)

[incremented by 0.1 ] is 1.330000

\section{Warnings}

line number: (11) : waming: 'poly_apply' : no return value line number: (22) : waming: conversion between different floating-point types line number: (56) : waming: 'argument' : different levels of indirection line number: (56) : waming: 'poly_apply' : different types for formal and actual parameter 1 line number: (56) : waming: 'argument' : different levels of indirection line number: (56) : warning: 'poly_apply' : different types for formal and actual parameter 2 line number: (56) : warning: ' $=$ ' : different levels of indirection line number: (56) : warning: conversion of near pointer to long integer 


\section{APPENDIX D}

\section{A Monomorphic Function Calls a Polymorphic Function}

\section{Executable Code}

1

2

3

4

5

6

11

12

13

14

15

16

17

18

19

20

21

22

23

24

25

26

27

28

29

30

31

32

33

34

35

36

37

38

39

40

41

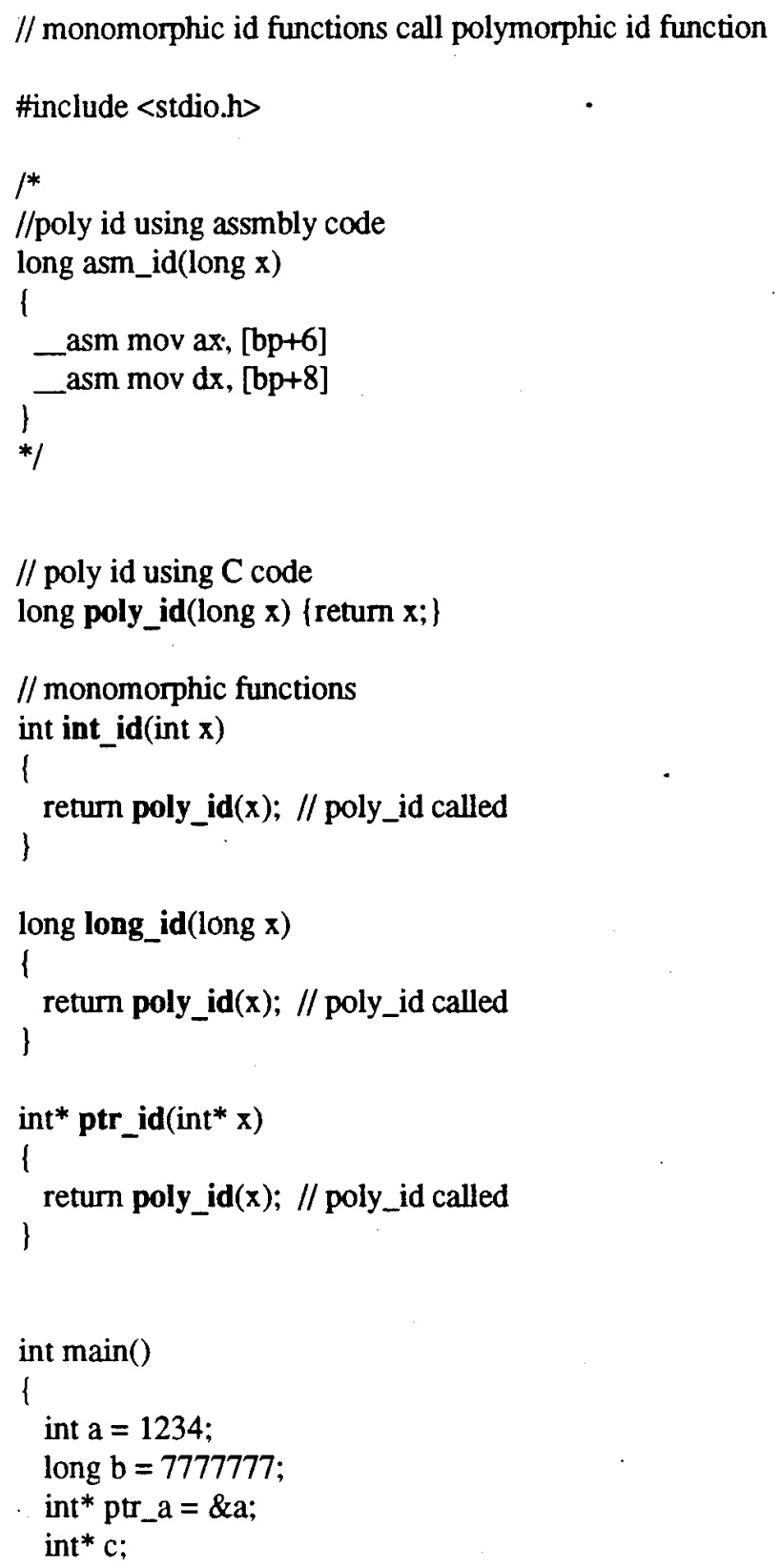


printf(" $n * * *$ testing id $* * * * * *(n \backslash n ")$;

printf(" \%d", int_id(a)); printf("V");

printf(" \%ld", long_id(b)); printf("v");

$\mathrm{c}=\mathrm{ptr}$ id $\left(\mathrm{ptr} \_\mathrm{a}\right)$;

printf("\%d", *c ); printf("v");

return 0 ;

49

50

\section{$\underline{\text { Test Results }}$}

$* * *$ testing id ******

1234

7777777

1234

\section{Warnings}

line number: (21): conversion between different integral types line number: (31): 'argument' : different levels of indirection line number: (31): 'poly_id' : different types for formal and actual parameter 1 line number: (31): 'return' : different levels of indirection line number: (31): conversion of near pointer to long integer 


\section{APPENDIX E}

\section{A Polymorphic Function calls a Polymorphic Function}

\section{Executable Code}

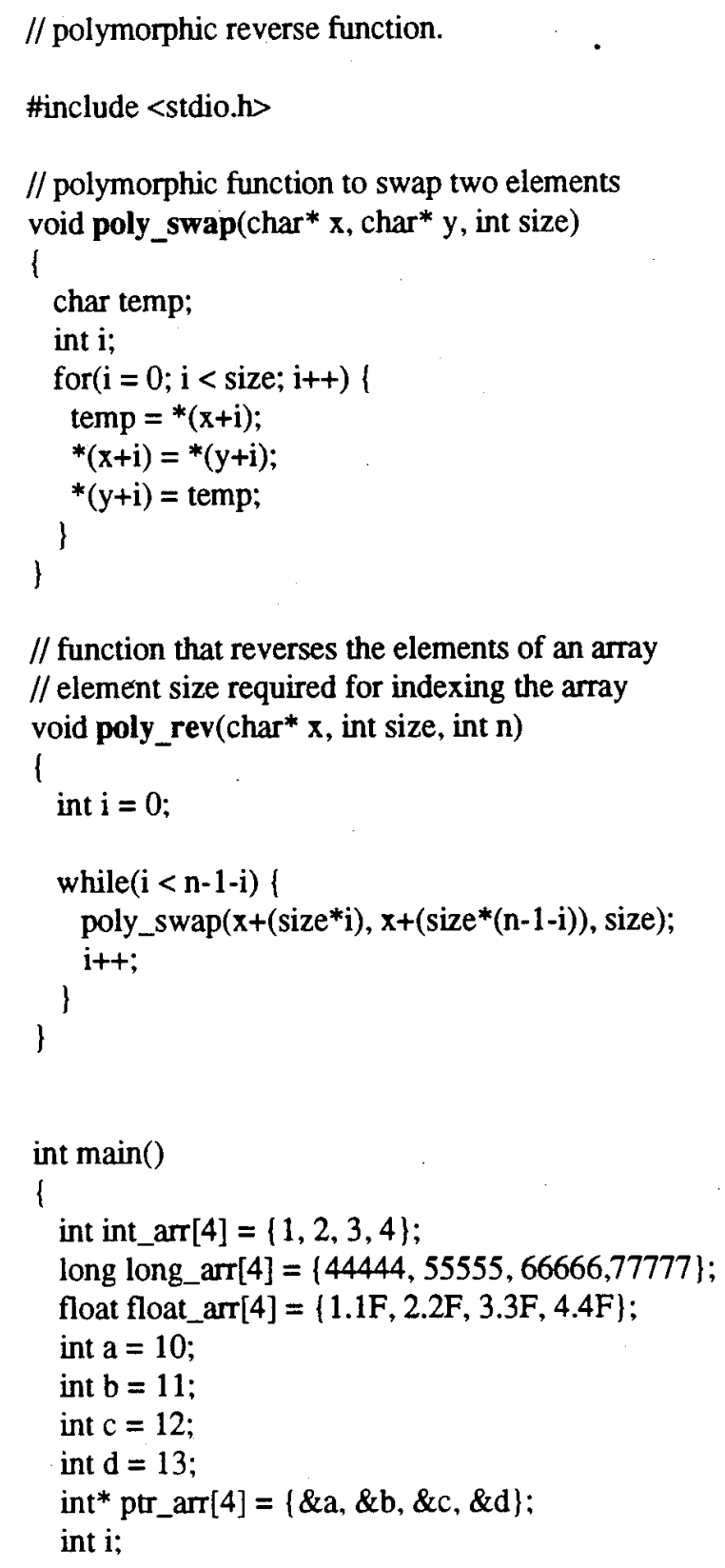




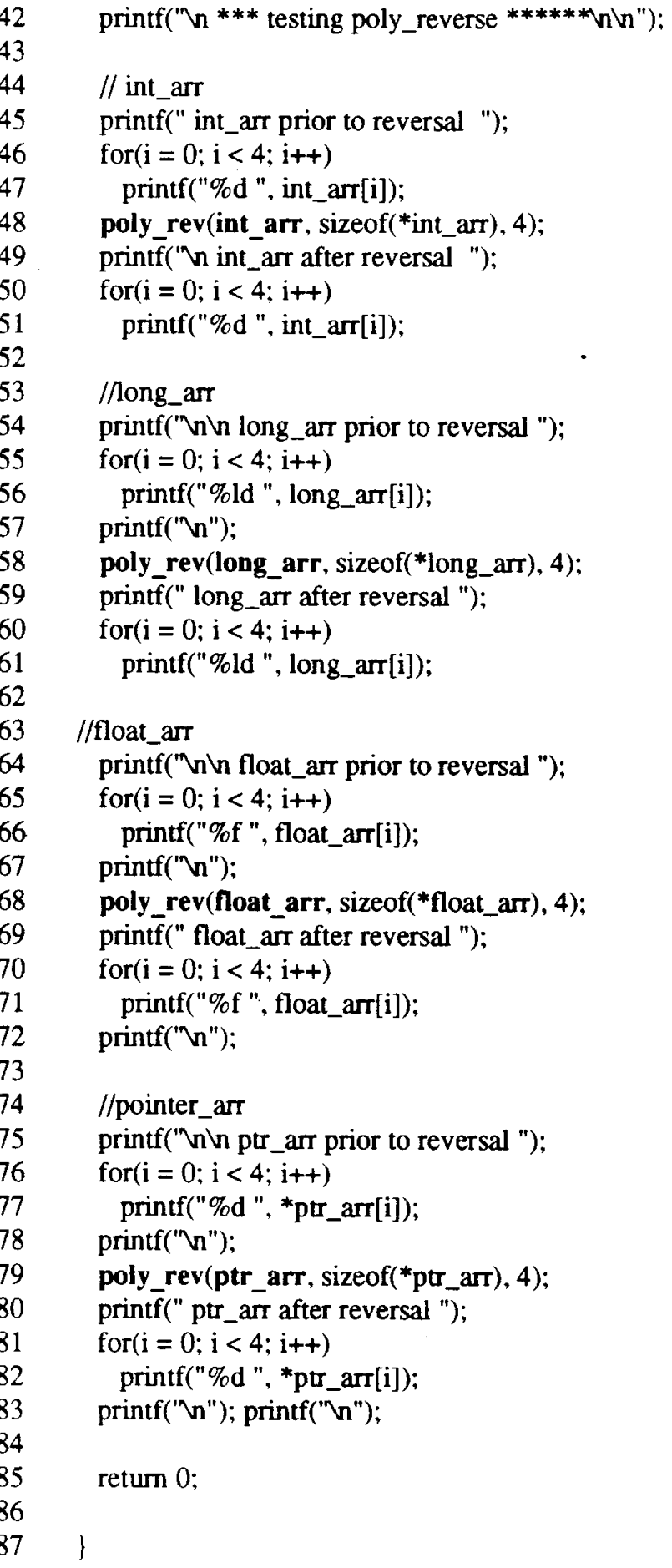




\section{Test Results}

*** testing poly_reverse $* * * * * *$

int_arr prior to reversal 1234

int_arr after reversal 4321

long_arr prior to reversal 44444555556666677777

long_arr after reversal 77777666665555544444

float_arr prior to reversal 1.1000002 .2000003 .3000004 .400000

float_arr after reversal 4.400000 3.300000 2.200000 1.100000

ptr_arr prior to reversal 10111213

ptr_arr after reversal 13121110

\section{Warnings}

line number: (48): 'argument' : indirection to different types

line number: (58): indirection to different types

line number: (68): indirection to different types

line number: (79): different levels of indirection .

line number: (79): different types for formal and actual parameter 1 



\section{REFERENCES}

[Bon95] Bonem, P., Towards an Implementation of Polymorphic C, Master's Thesis, Naval Postgraduate School, Monterey California, September 1995.

[Ler92] Leroy, S., "Unboxed Obejects and Polymorphic Typing", Proc. 19th ACM Symposiun on Principles of Programming Languages, January 1992.

[MDCB91] Morrison, R., Dearle, A., Connor, C., and Brown, A., "An Ad Hoc Approach to the Implementation of Polymorphism", ACM transactions on Programming Languages and Systems, Vol. 13, No. 3, July 1991.

[ShAp95] Shao, Z, and Appel, A., "A Typed-Based Compiler for Standard ML", Proc. 1995 Conf. on Programming Language Design and Implementation, June 1995.

[SmVo95] Smith, G., and Volpano, D., "An ML-style Polymorphic Type System for C", submitted for publication, 1995.

[Thi95] Thiemann, P., "Unboxed Values and Polymorphic Typing Revisited", Conference Record of ACM FPCA '95 Conference on Functional Programming Languages and Computer Architecture, June 1995. 


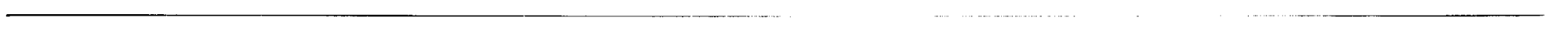

2

.

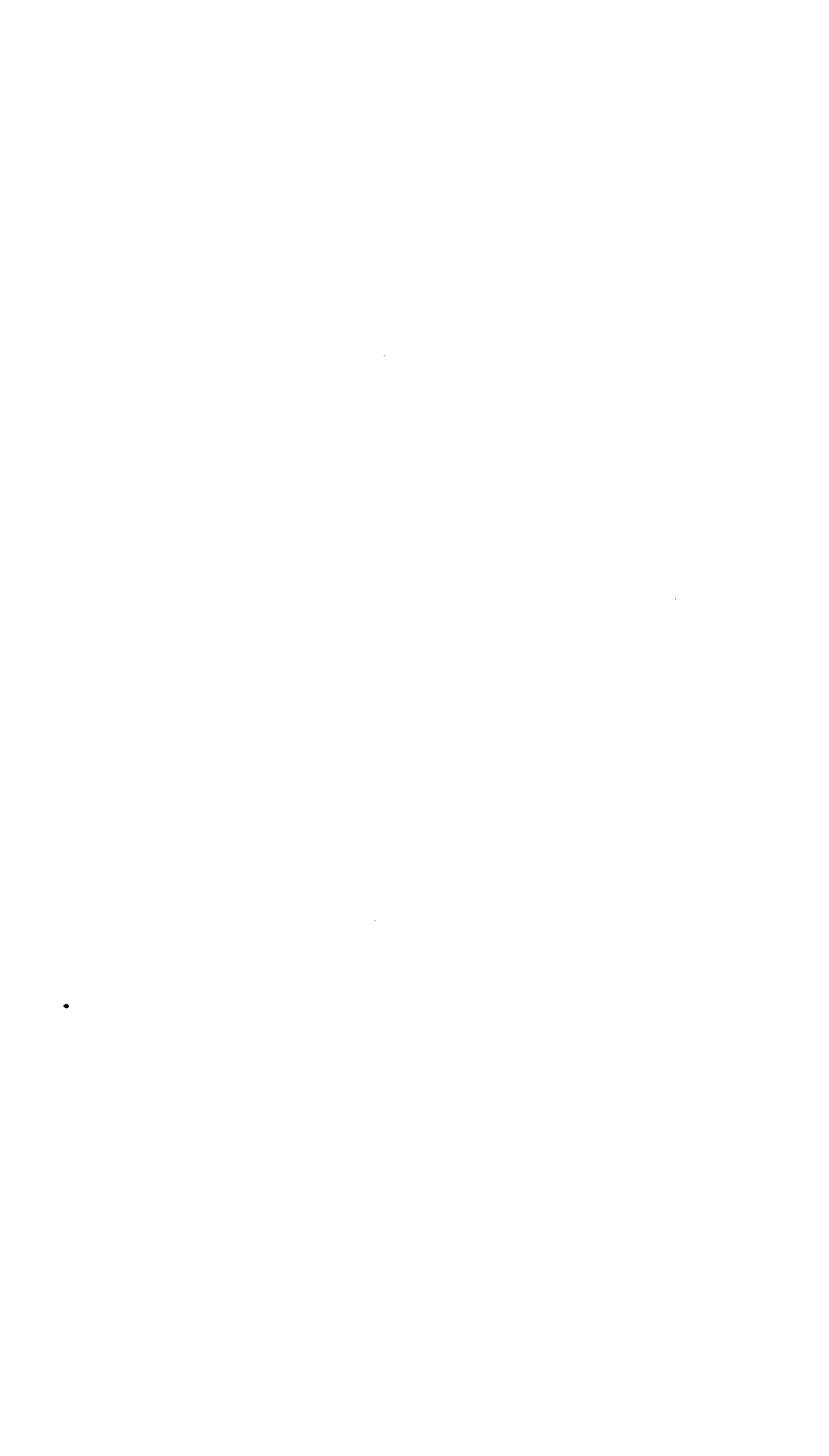




\section{DISTRIBUTION LIST}

Defense Technical Information Center

Cameron Station

Alexandria, VA 22314

2 copies

Library, Code 52

Naval Postgraduate School

Monterey, CA 93943

2 copies

Center for Naval Analyses

4401 Ford Avenue

Alexandria, VA 22302-0268

2 copies

Director of Research Administration

Code 012

Naval Postgraduate School

Monterey, CA 93943

1 copy

Dr. D. Volpano, Code CS/Vo

Computer Science Department

Naval Postgraduate School

Monterey, CA 93943

5 copies

Dr. G. Smith

School of Computer Science

Florida International University

University Park

Miami, FL 33199

1 copy

CDR Carl M. Pederson Jr., USN

PSC 78 BOX 346

APO AP 96326-0346

1 copy 